\title{
On Using Probabilistic Forwarding to Improve HEC-Based Data Forwarding in Opportunistic Networks ${ }^{\star}$
}

\author{
Ling-Jyh Chen ${ }^{1}$, Cheng-Long Tseng ${ }^{2}$, and Cheng-Fu Chou ${ }^{2}$ \\ ${ }^{1}$ Institute of Information Science, Academia Sinica \\ ${ }^{2}$ Department of Computer Science and Information Engineering, National Taiwan University
}

\begin{abstract}
In this paper, we propose the HEC-PF scheme, an enhancement of our previous $\mathrm{H}-\mathrm{EC}$ scheme for effective data forwarding in opportunistic networks. The enhanced scheme modifies the aggressive forwarding phase of the H-EC scheme by implementing a new Probabilistic Forwarding feature, which decides whether to forward a message to a newly encountered node based on the delivery probability. Using simulations as well as realistic network traces, we evaluate the performance of the proposed scheme in terms of delivery latency and completion ratio. The results show that the HEC-PF scheme outperforms the EC and $\mathrm{H}$-EC schemes in all test cases, and the performance gain is even more substantial when network connectivity is extremely poor. By varying the parameters of the HEC-PF scheme, we show that its completion ratio improves as the maximum forwarding distance or the hop distance considered when calculating the delivery probability increases. The effectiveness of the HEC-PF scheme makes it an ideal solution that goes a long way toward ensuring effective data delivery in opportunistic networks.
\end{abstract}

Keywords: Opportunistic Networks; Probabilistic Forwarding; Erasure Coding.

\section{Introduction}

An opportunistic network is a type of challenged network that has the following characteristics: (1) network contacts (i.e., communication opportunities) are intermittent; (2) an end-to-end path between the source and the destination has never existed; (3) disconnection and reconnection are common occurrences; and (4) the link performance is highly variable or extreme. Because of various disruptions and long delays, traditional MANET and Internet routing techniques can not be applied directly in opportunistic networks. Hence, with the development of numerous opportunistic networking applications, such as wireless sensor networks (WSN), underwater sensor networks (UWSN), pocket switched networks (PSN), people networks, and transportation networks, it is necessary to develop an effective data forwarding scheme that can better accommodate the various characteristics of opportunistic networks.

\footnotetext{
* This work was funded by the National Science Council under grant numbers NSC 95-2221-E001-025.
} 
Several data forwarding schemes have been proposed for opportunistic networks [5] [9] [12] [15] [16] [17] [19]. Such schemes can be divided into two main categories, replication-based and coding-based, according to their basic technical strategies. Replication-based routing schemes are the most popular design choice for existing opportunistic routing schemes. Basically, such schemes input multiple identical copies of data into the network, and rely on node mobility to forward the data to the destination [16]. A message is regarded as successfully delivered when at least one of the multiple copies is received by the destination. Intuitively, if the number of replicas in the network is sufficiently large, replication-based schemes should achieve the best delay performance (i.e., the shortest delivery latency) in opportunistic networks. However, the main drawback of this type of scheme is the tremendous traffic overhead associated with flooding a network with data replicas. As a result, when network resources are limited (e.g., the buffer space and network bandwidth), replication-based schemes will probably degrade performance reliability unless additional overhead reduction strategies are in place to alleviate the traffic overhead (e.g., [5] [9] [12] [15]).

For coding-based routing schemes, a message (or group of messages) is transformed into another format prior to transmission [17] [19]. The design principle of these schemes is to embed additional information (e.g., redundancy [17] or a decoding algorithm [19]) in the coded blocks such that the original message can be successfully reconstructed with only a certain number of the coded blocks. More precisely, unlike replication-based schemes, which rely on successful delivery of each individual data block, coding-based schemes consider a block successfully delivered when enough blocks are received to reconstruct the original data. As a result, coding-based schemes are usually more robust than replication-based schemes when a network's connectivity is extremely poor; however, they are less efficient when the network is fairly connected due to additional information embedded in the coded blocks.

In [7], Chen et al. proposed a hybrid scheme, called H-EC, which combines the strengths of replication-based schemes and coding-based schemes by integrating their respective aggressive forwarding technique and erasure coding technique. Hence, the $\mathrm{H}$-EC scheme not only remains robust when the network connectivity is extremely poor, but also performs efficiently when the network is fairly connected. Even so, the performance of the scheme depends to a large extent on the message scheduling algorithm used in the aggressive forwarding phase. This aspect has not been discussed extensively and algorithms have only been implemented in a First-Come-First-Served (FCFS) fashion [3]. Thus, in this paper, we investigate effective message scheduling algorithms that consider both the frequency and volume of contacts in a network's history, and thereby substantially improve the data forwarding performance of the $\mathrm{H}$-EC scheme in opportunistic networks.

The remainder of the paper is organized as follows. In Section 2 we review related works on opportunistic routing, and provide an overview of the H-EC scheme. In Section 3 we propose the HEC-PF scheme, which employs the Probabilistic Forwarding feature in the aggressive forwarding phase of the H-EC scheme. Section 4 presents a comprehensive set of simulation results for various opportunistic network scenarios; the results are also analyzed and explained in detail. We then present our conclusions in Section 5 


\section{Related Work and Overview of the H-EC Scheme}

\subsection{Related Work}

Routing in an opportunistic network is challenging and completely different to conventional network routing methods. In opportunistic networks, an ideal routing scheme has to provide reliable data delivery, even when the network's connectivity is intermittent or when an end-to-end path is temporally unavailable. Moreover, since "contacts" in an opportunistic network may appear arbitrarily without prior information, scheduled optimal routing methods (e.g., linear programming-based routing [11]) and mobile relay approaches (e.g., Message Ferrying [20]) cannot be applied.

Currently, replication is the most popular design choice for opportunistic routing schemes. For instance, the Epidemic Routing scheme [16] sends identical copies of a message simultaneously over multiple paths to mitigate the effects of a single path failure, thereby increasing the possibility of successful message delivery. However, flooding a network with duplicate data tends to be very costly in terms of traffic overhead and energy consumption. To address the excess traffic overhead incurred by flooding replicate data, the Controlled Flooding proposed in [9] reduces the flooding cost while maintaining reliable message delivery.

Node mobility also impacts on the effectiveness of opportunistic routing schemes. When network mobility differs from the well-known random way-point mobility model, the overhead of epidemic- and/or flooding-based routing schemes can be further reduced by considering node mobility. For instance, the PRoPHET scheme [14] calculates the delivery predictability from a node to a particular destination node based on the observed contact history, and forwards a message to its neighboring node if and only if that neighbor node has a higher delivery predictability value. Leguay et al. [12] revised this scheme by taking a node's mobility pattern into account, i.e., a message is forwarded to a neighbor node if and only if the neighbor node has a mobility pattern similar to that of the destination node. The approach described in [12] shows that the revised mobility pattern-based scheme is more effective than previous methods.

Another class of opportunistic network routing schemes is based on encoding techniques, which transform a message into another format prior to transmission. For example, the integration of network coding and epidemic routing techniques has been proposed to reduce the number of transmissions required in a network [19], while [17] proposes combining erasure coding and the simple replication-based routing method to improve the data delivery in the worst delay performance cases in opportunistic networks. Following [17], an Estimation-based Erasure-Coding (EBEC) routing scheme has been proposed to adapt the delivery of erasure coded blocks using the Average Contact Frequency (ACF) estimate [13]. Moreover, [7] proposes a hybrid scheme that combines the strength of erasure coding and the advantages of Aggressive Forwarding, so that it is robust in worst delay performance cases, and performs efficiently in very small delay performance cases.

\subsection{H-EC: An Overview}

We now present an overview of erasure coding schemes and the H-EC scheme [7], which is a hybrid data forwarding scheme based on erasure coding. 


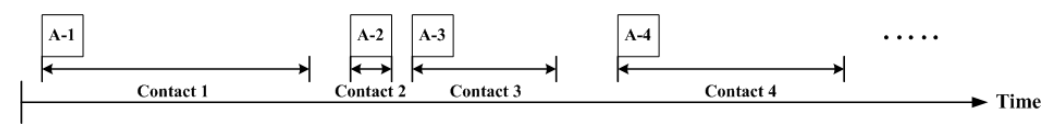

Fig. 1. The erasure coding-based data forwarding algorithm (EC). In this figure, one erasure coded block (A) is split equally among four relays $(n=4)$.

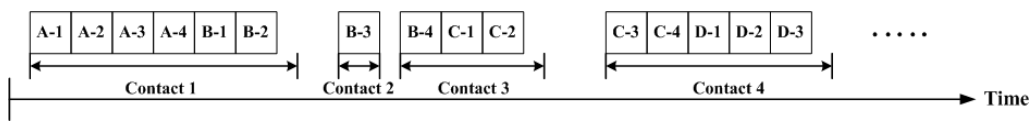

Fig. 2. The A-EC scheme, i.e., EC with aggressive forwarding. In this figure, four erasure coded blocks (A,B,C,D) are transmitted, with $n=4$.

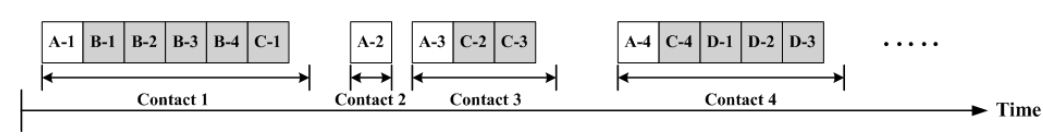

Fig. 3. The H-EC scheme. Under the scheme, two copies of four erasure coded blocks (A,B,C,D) are transmitted: the first copy of EC blocks (the white blocks) is sent using the EC algorithm, and the second copy (the gray blocks) is sent using the A-EC algorithm in the residual contact time. Each coded block is split into 4 equal-sized sub-blocks $(n=4)$.

By adding redundancy, erasure coding improves the fault-tolerance without the overhead of strict replication of the original data [18]. In a generic erasure coding scheme, given a message of size $M$ bytes, a replication factor of erasure coding $r$, and a coded message fragmented into several blocks of equal-sized bytes $b$, the number of coded blocks can be derived by $N=\frac{M \times r}{b}$. Moreover, this message can be successfully reconstructed as long as $\frac{1}{r}$ of the coded blocks is received.

An erasure code-based (EC) forwarding algorithm is proposed in [17]. In this scheme, (see Fig. 1), the erasure coded blocks are split equally among $n$ relays, which are only allowed to send messages to the destination directly (this is the well-known "two-hop" scenario used in [8]). Each relay forwards the same number of coded blocks, and there are no duplicates in a relay.

As reported in [17], the EC scheme achieves the most effective worst-case delay performance with a fixed amount of overhead. However, the drawback of the scheme is that it can not provide a good very small delay performance compared to other popular replication-based approaches. The reason for this inefficiency is because, if most network contacts are much longer than the required time, the EC scheme tends to waste the residual contact period; hence, it is ineffective, as illustrated in Fig.11

To resolve the above problem, [7] proposed an enhanced scheme called A-EC, i.e., EC with an aggressive forwarding feature, as shown in Fig. 2. Under this scheme, the source sends as many coded blocks as possible during each contact period. It has been shown that the A-EC scheme is better able to utilize network contacts; thus, it can

\footnotetext{
${ }^{1}$ For simplicity, following [17], we assume $N=n$ for all cases.
} 
be expected to outperform the EC scheme for very small delay performance cases. However, for worst delay performance cases, A-EC yields a poor delivery ratio and/or a very large delivery delay when black-holes 2 are present in the network [7].

Taking advantage of the strengths of the EC and A-EC schemes to achieve better message delivery performance in both worst delay performance and very small delay performance cases, Chen et al. [7] proposed a hybrid scheme, called H-EC, which is illustrated in Fig. 3. In the H-EC scheme, two copies of EC blocks (constructed by the erasure coding and replication techniques described earlier) are transmitted by the sender. The first copy of EC blocks is sent in a similar way to the method used to send blocks in the original EC scheme (the white blocks in Fig. 3], while the second copy is sent using aggressive forwarding during the residual contact time after sending the first EC block (the gray blocks in Fig. 3). For general opportunistic network scenarios (i.e., without black-hole nodes), the H-EC scheme utilizes each contact opportunity better because of the aggressive forwarding feature; however, if black-hole nodes are present in the network, the scheme's performance is similar to that of the EC scheme, which achieves better forwarding in worst delay performance cases.

The performance of the H-EC scheme depends to a large extent on the message scheduling algorithm used in the aggressive forwarding phase, which is not discussed in [7]. However, in this paper, we assess the impact of probabilistic message scheduling algorithms on the performance of $\mathrm{H}$-EC routing. To this end, we propose an extension of the H-EC scheme, called HEC-PF, in the following section.

\section{HEC-PF: H-EC with Probabilistic Forwarding}

In this section, we propose a message scheduling algorithm, called Probabilistic Forwarding, for the aggressive forwarding phase of the $\mathrm{H}$-EC scheme. The resulting scheme is called HEC-PF. Unlike the H-EC scheme [7], the HEC-PF scheme does NOT enter the aggressive forwarding phase unless a newly encountered node has a higher likelihood of successfully forwarding the message to the destination node than the current node. We describe the HEC-PF scheme in the following sub-sections.

\subsection{Delivery Probability}

The key issue for the HEC-PF scheme is how to estimate the likelihood of successfully transmitting a message from a given node to the destination node. Similar to the PRoPHET scheme [14], the HEC-PF scheme estimates the delivery probability based on the observed contact history. However, unlike PRoPHET, the HEC-PF scheme considers the contact frequency in the history as well as the contact volume, which represents the proportion of time that the two nodes are in contact in the last $T$ time units. More specifically, if there are $K$ nodes in the network, we denote the $i$-th node as $X_{i}$, the $j$-th node as $X_{j}$, the aggregate contact volume between the node pair $X_{i}$ and $X_{j}$ in the last $T$ time units as $t_{X_{i}, X_{j}}$, and the delivery probability for the node pair $X_{i}$ and $X_{j}$ with a distance of at most $k$-hops as $P_{X_{i}, X_{j}}^{k}$. The one-hop delivery probability from the

\footnotetext{
${ }^{2}$ A node in a network is called a black-hole if it is either unreliable (e.g., it has very limited battery power and/or buffer size) or it hardly moves towards the destination [7].
} 
source node $\left(X_{S}\right)$ to the destination node $\left(X_{D}\right)$ is given by the ratio of the aggregate contact volume over the overall contact volume? as shown in Eq.1.

$$
P_{X_{S}, X_{D}}^{1}=\frac{t_{X_{S}, X_{D}}}{\sum_{i=1}^{K} t_{X_{S}, X_{i}}}
$$

In addition, the two-hop delivery probability, $P_{X_{S}, X_{D}}^{2}$, can be derived by Eq. 2. The equation is comprised of three components: the scaling constant, $\omega_{2} \in[0 \ldots 1]$, which decides the impact of two-hop message transfer on the overall delivery probability; the likelihood value, $1-P_{X_{S}, X_{D}}^{1}$, which is the probability that a message can not be transmitted directly from node $X_{S}$ to node $X_{D}$ (i.e., it is impossible to complete the message delivery in one hop); and the sum of the two-hop transitive delivery probability based on the transitive property, i.e., if node $X_{S}$ frequently encounters node $X_{i}$, and node $X_{i}$ frequently encounters node $X_{D}$, then $X_{i}$ is a good candidate relay node for forwarding messages from node $X_{S}$ to node $X_{D}$.

$$
P_{X_{S}, X_{D}}^{2}=\omega_{2}\left(1-P_{X_{S}, X_{D}}^{1}\right) \sum_{\substack{1 \leq i<K \\ i \neq S, i \neq D}}\left(P_{X_{S}, X_{i}}^{1} P_{X_{i}, X_{D}}^{1}\right)
$$

Similarly, the three-hop delivery probability can be estimated by Eq. 3 and the $k$ hop delivery probability can be derived by Eq. 4. Finally, the delivery probability of transferring a message from node $X_{S}$ to node $X_{D}$ is given by summing the delivery probabilities of all $k$ cases, as shown by Eq. 5

$$
\begin{aligned}
& P_{X_{S}, X_{D}}^{3}=\omega_{3}\left(1-P_{X_{S}, X_{D}}^{1}-P_{X_{S}, X_{D}}^{2}\right) \times \sum_{\substack{1 \leq i, j \leq K \\
i \neq S, i \neq D \\
j \neq S, j \neq D}}\left(P_{X_{S}, X_{1}}^{1} P_{X_{1}, X_{2}}^{1} P_{X_{2}, X_{D}}^{1}\right) \\
& P_{X_{S}, X_{D}}^{k}=\omega_{k}\left(1-\sum_{i=1}^{k-1} P_{X_{S}, X_{D}}^{i}\right) \times \\
& \sum_{\substack{1 \leq a_{i} \leq K, a_{i} \neq S, a_{i} \neq D, a_{i} \neq a_{j} ; \forall 1 \leq i, j \leq k}}\left(P_{X_{S}, X_{a_{1}}}^{1}\left(\prod_{i=1}^{k-2} P_{X_{a_{i}}, X_{a_{i+1}}}^{1}\right) P_{X_{a_{k-1}}, X_{D}}^{1}\right) \\
& P_{X_{S}, X_{D}}=\sum_{i=1}^{k} P_{X_{S}, X_{D}}^{i}
\end{aligned}
$$

Note that deciding an adequate value for $k$ involves a tradeoff. On the one hand, the larger the value of $k$, the more accurately we can approximate the delivery probability; on the other hand, using a large $k$ is very likely to incur an enormous storage and computation overhead. An ideal solution would be able to adapt its $k$ settings to the properties of the network. For simplicity, we set $k$ to a constant value in the simulations, and defer a detailed discussion and evaluation of this issue to a future work.

\footnotetext{
${ }^{3}$ If $i==j, t_{X_{i}, X_{j}}=0$.
} 


\subsection{Probabilistic Forwarding}

We now describe the HEC-PF scheme in detail. As mentioned earlier, the scheme incorporates a new Probabilistic Forwarding feature that decides whether to forward a message to a newly encountered node based on the delivery probability estimate, rather than on a first-come-first-served basis. The HEC-PF scheme is better able to deliver a message successfully because it tends to utilize relay nodes that have higher delivery probabilities.

More precisely, suppose that node $X_{i}$ holds a message from the source node $X_{S}$ for transmission to the destination node $X_{D}$, and the message has not been relayed more than $H$ times so far. There are two cases where $X_{i}$ could accidentally encounter another node $X_{j}$ (assuming $X_{j} \neq X_{D}$ ) if there is time remaining after $X_{i}$ sends out one block of the first copy of the EC blocks. First, if $X_{i}$ is the source node, $X_{i}$ must make a decision about whether to enter the aggressive forwarding phase by comparing the two delivery probabilities, $P_{X_{i}, X_{D}}$ and $P_{X_{j}, X_{D}}$. If $P_{X_{i}, X_{D}}>P_{X_{j}, X_{D}}, X_{i}$ will enter the aggressive forwarding phase in the same way as the original H-EC scheme; otherwise, it will follow the EC scheme and not enter the aggressive forwarding phase.

In the second case (i.e., $X_{i}$ is not the source node), $X_{i}$ first checks whether the nextto-be-sent block was sent by the source node during the aggressive forwarding phase (i.e., whether it belongs to the second copy of the EC blocks). If it was, $X_{i}$ forwards the block to $X_{j}$ as long as $X_{j}$ has a higher delivery probability than $X_{i}$ of successfully forwarding the block to $X_{D}$ (i.e., $P_{X_{j}, X_{D}}>P_{X_{i}, X_{D}}$ ); otherwise, the block belongs to the first-copy of the EC blocks, and $X_{i}$ forwards it to $X_{j}$ automatically, i.e., without checking the delivery probability.

\section{Evaluation}

We use a set of simulations to evaluate the delay and completion ratio performance of the HEC-PF scheme in opportunistic networks. We implement the HEC-PF scheme by extending the H-EC codes [3] and run simulations in DTNSIM [2], a Java-based DTN simulator. Similar to the scenarios described in [17], messages are generated at a Constant Bit Rate (CBR) of 12 messages per day for 160 days (i.e., $M=12 \times 160=$ $1,920)$, and the size of each message is 1,000 bytes. For all EC based schemes, the code block size $b$ is set to 125 bytes, and the other two parameters, $r$ and $n$, are set to 2 and 16 respectively. In addition, under the HEC-PF scheme, the sliding time window $T$ (used to estimate the delivery probability) is set to 1,000 seconds; and the scaling constant $\omega_{i}$ is set to 0.25 for $i=2 \ldots 5$. The simulation results represent the average performance of 200 simulation runs. In each run the source and destination pair was randomly selected from all the participating nodes.

We evaluate three network scenarios. The first is generated according to the powerlaw distribution by setting both the inter-contact time and the contact duration of the networks as power-law distributed random variables with coefficient equal to 0.6 (following [10]). There are 34 participating nodes. The other two scenarios are based on realistic campus wireless network traces, namely, the iMote [1] and UCSD [4] traces, which are publicly available for research purposes. Table 1 outlines the basic properties of the three network scenarios. 
Table 1. The properties of the three network scenarios

\begin{tabular}{|c|c|c|c|}
\hline Trace Name & Power-Law & iMote & UCSD \\
\hline Device & N/A & iMote & PDA \\
\hline Network Type & N/A & Bluetooth & WiFi \\
\hline Duration (days) & 16 & 3 & 77 \\
\hline Devices participating & 34 & 274 & 273 \\
\hline Number of contacts & 25,959 & 28,217 & 195,364 \\
\hline Avg \# Contacts/pair/day & 2.89205 & 0.12574 & 0.06834 \\
\hline
\end{tabular}

The UCSD trace is client-based and records the availability of WiFi-based access points (APs) for each participating portable device (e.g., PDAs and laptops) on the UCSD campus. Similar to [6] [7] [10], we assume that two participating devices in ad hoc mode encounter a communication opportunity (i.e., a network contact) if and only if they are both associated with the same AP at the same time.

The iMote trace is a human mobility trace collected at the 2005 IEEE Infocom conference. It was aggregated from 41 Bluetooth-based iMote devices, which were distributed to the student attendees for the duration of the 3-day conference. Each iMote device was pre-configured to periodically broadcast query packets to find other Bluetooth devices within range, and record the devices that responded to the queries. In addition to the distributed iMote devices, another 233 devices were recorded in the trace. They may have been other Bluetooth-enabled devices used during the conference. For simplicity, we assume there is a network contact between two Bluetooth devices if there exists a query-and-response interaction between them.

\subsection{Evaluation I: Two-Hop Scenario}

In the first set of simulations, we evaluate the delay performance of the HEC-PF scheme for message delivery in opportunistic networks. Three network scenarios are examined via simulations, with $H$ set to 2 (i.e., the conventional two-hop scenario used in [7] [17]). The $k$ parameter of the HEC-PF scheme is set to 2 (i.e., we consider the transitive property of message delivery with a distance of up to two hops). Fig. 4 depicts the average data latency distribution results in Complementary CDF (CCDF) curves.

From Fig. 4, we observe that the HEC-PF scheme consistently outperforms the H-EC and EC schemes in all test scenarios. The reason is that HEC-PF employs the Probabilistic Forwarding feature, which decides whether to forward a message to a newly encountered node based on the delivery probability estimates, rather than on the firstcome-first-served (FCFS) basis used in the original H-EC scheme. As a result, HEC-PF is better able to make use of nodes that are more likely to encounter the destination node, and thereby achieve better message delivery performance.

We also observe that the completion ratio, i.e., the percentage of messages successfully transmitted before the end of the simulation, degrades as the network connectivity (i.e., the average number of network contacts per node pair, per day) decreases. For example, although the HEC-PF scheme achieves approximately $99 \%$ completion ratio in the Power-Law scenario, it can only achieve about $80 \%$ in the iMote scenario and 


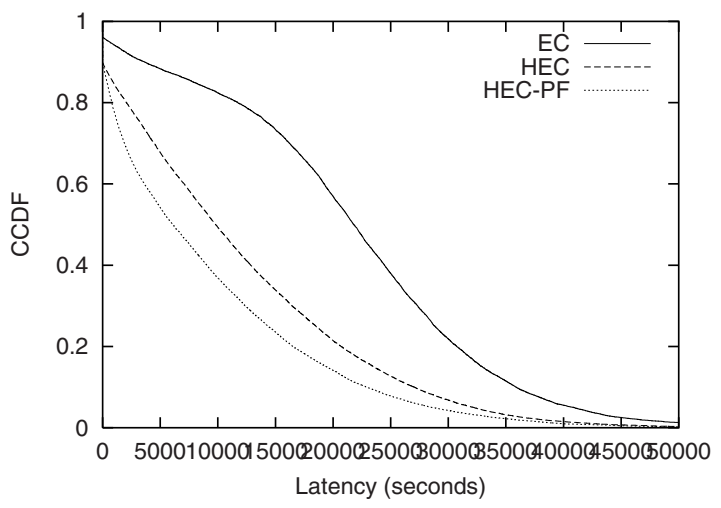

(a) Power-Law Scenario

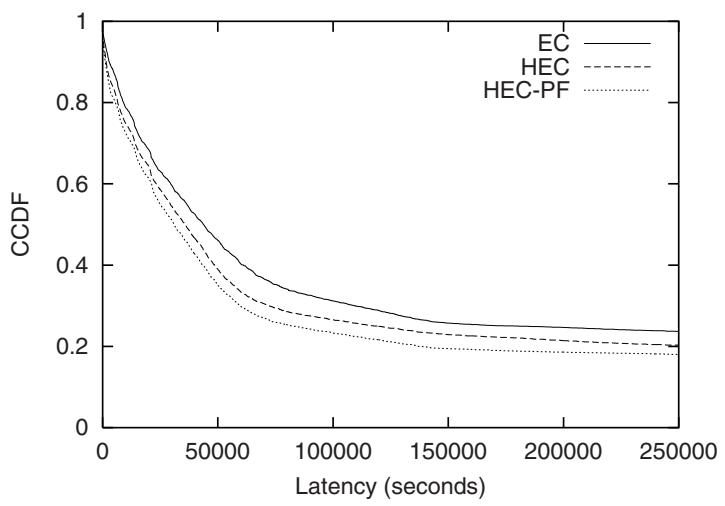

(b) iMote Scenario

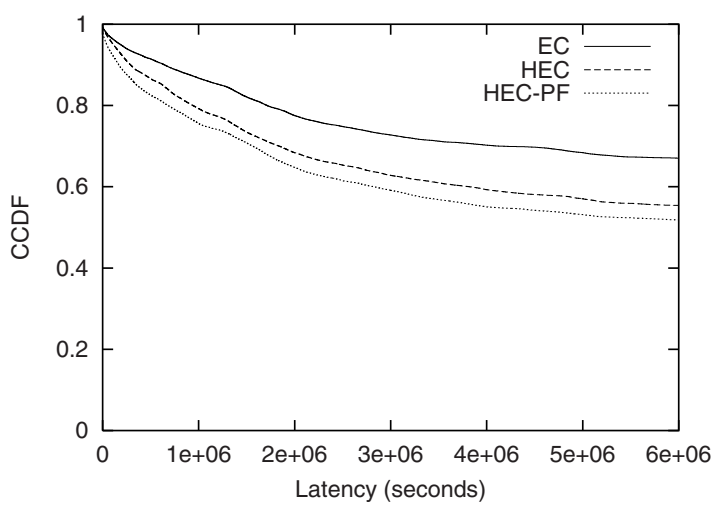

(c) UCSD Scenario

Fig. 4. Distribution (CCDF) of average latency performance of the EC, H-EC, and HEC-PF schemes $(N=16, r=2, k=2$, and $H=2)$ 
$50 \%$ in the UCSD scenario. The reason is that the two-hop forwarding strategy is not sufficient to deliver all messages in the limited simulation time because the network's connectivity is poor. Another observation is that the performance gain of the HEC-PF scheme (compared to the H-EC scheme) is not significant. This implies that the calculation of the delivery probability (with $k=2$ in this case) cannot provide sufficient information to make a decision on whether to forward a message in the aggressive forwarding phase of the HEC-PF scheme. The findings motivate us to investigate the impact of the HEC-PF parameters on the performance of message delivery in opportunistic networks. We present the evaluation in the next subsection.

\subsection{Evaluation II: Variable $\boldsymbol{H}$ Scenarios}

In the second set of evaluations, we evaluate the performance of the HEC-PF scheme in the UCSD scenario with various maximum forwarding distance settings $(H=2,3,4,5)$. Recall that the connectivity of this scheme is deemed to be poor. Similar to the previous evaluation, we set the $k$ parameter of the HEC-PF scheme to 2. The average data latency distribution results are shown as CCDF curves in Fig.5

The results in Fig. 5 show that the CCDF curve falls as the value of $H$ increases. More specifically, at the end of the simulation, the HEC-PF scheme improves the completion ratio from $48 \%$ to $62 \%$ as $H$ is increased from 2 to 5 . The reason is that the larger the setting of $H$, the greater the likelihood that a message will be delivered to the destination node eventually. Of course, when a large $H$ is employed in the HEC-PF scheme, an extensive amount of one-hop data forwarding is required; thus, more energy will be consumed in the network (as shown in Table 2 the transmission overhead of the HEC-PF scheme increases substantially as the value of $H$ increases). Again, an ideal solution for HEC-PF should adapt its $H$ value to the properties of the network in order to compensate for the above tradeoff. We defer a detailed discussion and evaluation of this issue to a future work.

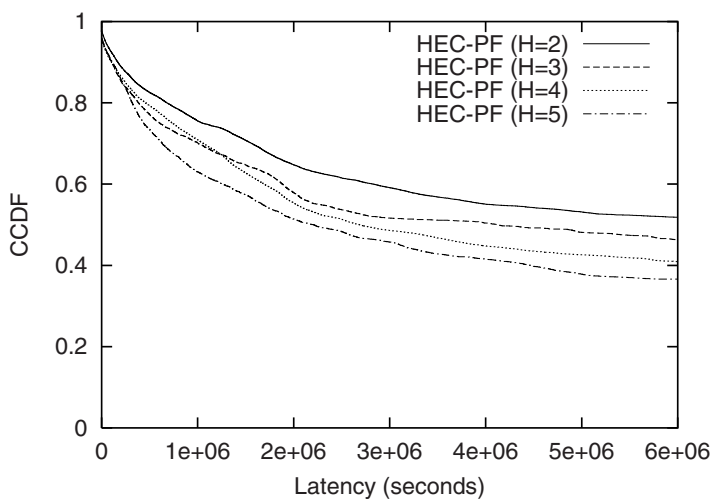

Fig. 5. Distribution (CCDF) of average latency performance of the HEC-PF scheme in the UCSD scenario with various $H$ settings $(N=16, r=2$, and $k=2$ ) 


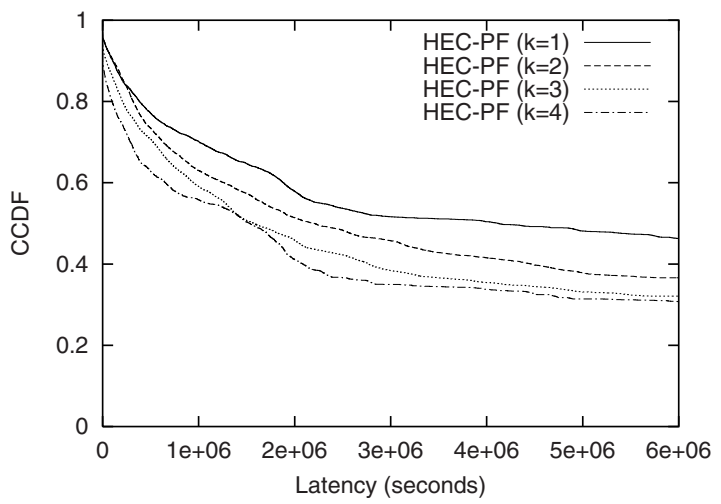

Fig. 6. Distribution (CCDF) of average latency performance of the HEC-PF scheme in the UCSD scenario with various $k$ settings $(N=16, r=2$, and $H=5$ )

Table 2. Transmission overhead of HEC-PF Table 3. Transmission overhead of HEC-PF with various $H$ values. (Unit: MBytes) with various $k$ values. (Unit: MBytes)

\begin{tabular}{|c|c|c|c|c|}
\hline Scenario & $H=2$ & $H=3$ & $H=4$ & $H=5$ \\
\hline PowerLaw & 7,527 & 9,747 & 13,088 & 15,455 \\
\hline iMote & 2,324 & 3,200 & 4,020 & 4,818 \\
\hline UCSD & 5,048 & 10,327 & 13,857 & 17,309 \\
\hline
\end{tabular}

\begin{tabular}{|c|c|c|c|c|}
\hline Scenario & $k=1$ & $k=2$ & $k=3$ & $k=4$ \\
\hline PowerLaw & 15,062 & 15,455 & 15,899 & 16,234 \\
\hline iMote & 4,523 & 4,818 & 4,897 & 4,950 \\
\hline UCSD & 15,668 & 17,309 & 21,197 & 22,018 \\
\hline
\end{tabular}

\subsection{Evaluation III: Variable $k$ Scenarios}

Here, we evaluate the performance of the HEC-PF scheme in the poorly connected network scenario (i.e., the UCSD scenario) with various $k$ values $(k=1 \ldots 5)$. The configurations of the evaluation are the same as previously, except that the maximum forwarding distance $H$ is set to 5. Fig. 6depicts the average data latency distribution results in CCDF curves.

From Fig. 6 it is evident that the CCDF curve falls as $k$ increases, which means that, given the same $H$ setting, the completion ratio of message delivery increases as the hop distance (used to estimate the delivery probability) increases; however, the transmission overhead of the HEC-PF scheme only increases moderately as the value of $k$ increases, as shown in Table 3 . The figure also shows that the performance gain of the completion ratio decreases as $k$ increases, which indicates that the completion ratio tends to converge. It is also worth noting that $k$ must be configured less than $H$ when estimating the delivery probability, since it is impossible to deliver a message successfully with a hop distance larger than the maximum forwarding distance.

\section{Conclusion}

We have proposed a scheme called HEC-PF that extends the basic H-EC scheme for data forwarding in opportunistic networks. The HEC-PF scheme incorporates a novel feature, called Probabilistic Forwarding feature, which decides whether to forward a message to a newly encountered node based on the delivery probability estimate in the aggressive forwarding phase. As a result, the scheme can find relays that are more likely 
to transmit a message to the destination node based on the historical record of network contacts. Using simulations as well as realistic network traces, we evaluated the performance of the proposed scheme in terms of its delivery latency and completion ratio. The results show that it outperforms the EC and H-EC schemes in all test cases, and the performance gain is even more significant when the network connectivity is extremely poor. In addition, by varying the values of the parameters of the proposed scheme, we have shown that its completion ratio improves as the maximum forwarding distance or the considered hop distance of the delivery probability increases. Work on developing mechanisms to determine the HEC-PF parameters that can adapt to the properties of a network is still ongoing. We will report on the results in the near future.

\section{References}

1. Crawdad project. http:// crawdad.cs.dartmouth.edu/

2. Delay tolerant network simulator. http: / /www.dtnrg.org/code/dtnsim.tgz

3. H-ec module for dtnsim simulator. http://nrl.iis.sinica.edu.tw/DTN/download/

4. Ucsd wireless topology discovery project. http://sysnet.ucsd.edu/wtd/

5. Burgess, J., Gallagher, B., Jensen, D., Levine, B.N.: Maxprop: Routing for vehicle-based disruption-tolerant networking. In: IEEE Infocom (2006)

6. Chaintreau, A., Hui, P., Crowcroft, J., Diot, C., Gass, R., Scott, J.: Impact of human mobility on the design of opportunistic forwarding algorithms. In: IEEE Infocom (2006)

7. Chen, L.-J., Yu, C.-H., Sun, T., Chen, Y.-C., Chu, H.h.: A hybrid routing approach for opportunistic networks. In: ACM CHANTS (2006)

8. Grossglauser, M., Tse, D.: Mobility increases the capacity of ad-hoc wireless networks. In: IEEE Infocom (2001)

9. Harras, K.A., Almeroth, K.C., Belding-Royer, E.M.: Delay tolerant mobile networks (dtmns): Controlled flooding in sparse mobile networks. In: IFIP Networking (2005)

10. Hui, P., Chaintreau, A., Scott, J., Gass, R., Crowcroft, J., Diot, C.: Pocket switched networks and human mobility in conference environments. In: ACM WDTN (2005)

11. Jain, S., Fall, K., Patra, R.: Routing in a delay tolerant network. In: ACM SIGCOMM (2004)

12. Leguay, J., Friedman, T., Conan, V.: Dtn routing in a mobility pattern space. In: ACM WDTN (2005)

13. Liao, Y., Tan, K., Zhang, Z., Gao, L.: Estimation based erasure-coding routing in delay tolerant networks. In: IWCMC (2006)

14. Lindgren, A., Doria, A.: Probabilistic routing protocol for intermittently connected networks. Technical report, draft-lindgren-dtnrg-prophet-01.txt, IETF Internet draft (July 2005)

15. Lindgren, A., Doria, A., Schelen, O.: Probabilistic routing in intermittently connected networks. ACM Mobile Computing and Communications Review 7(3), 19-20 (2003)

16. Vahdat, A., Becker, D.: Epidemic routing for partially-connected ad hoc networks. Technical Report CS-2000-06, Duke University (2000)

17. Wang, Y., Jain, S., Martonosi, M., Fall, K.: Erasure coding based routing for opportunistic networks. In: ACM WDTN (2005)

18. Weatherspoon, H., Kubiatowicz, J.D.: Erasure coding vs. replication: A quantitative comparison. In: IEEE IPTPS (March 2002)

19. Widmer, J., Boudec, J.-Y.L.: Network coding for efficient communication in extreme networks. In: ACM WDTN (2005)

20. Zhao, W., Ammar, M., Zegura, E.: A message ferrying approach for data delivery in sparse mobile ad hoc networks. In: ACM MobiHoc (2004) 